\title{
Comunidades Bentónicas Presentes en Sitios de Buceo en la Reserva Marina EI Pelado
}

\section{Benthic Communities Present in Diving Sites in the EI Pelado Marine Reserve}

Maritza Cárdenas-Calle 1,2,*, Miguel Triviño ${ }^{1}$, Ginatta Giovanni ${ }^{3}$, Mauricio Velásquez ${ }^{4}$

\section{INFORMACIÓN DEL ARTÍCULO}

Fecha de recepción: 13 de Marzo de 2018.

Fecha de aceptación: 02 de Julio de 2018

Bioelite, División Ambiental, Bosques El Salado Mz. 301 S2B, Guayaquil, Ecuador.

${ }^{2}$ Universidad de Guayaquil, Facultad de Ingeniería Química, Ciudadela Universitaria Salvador Allende.Av. Delta y Av. Kennedy, Guayaquil, Ecuador.

3 Corpei, Av. De las Américas N406, Centro de Convenciones, Oficina 1 , Planta Baja Guayaquil, Ecuador.

\footnotetext{
4 Banco de Desarrollo de América Latina (CAF), Unidad de Negocios Verdes. Dirección de Sostenibilidad, Inclusión y Cambio Climático. Av. 12 de Octubre N.24-562 y Cordero Edificio World Trade Center, Torre A, Av. 12 de Octubre piso 13, Quito, Ecuador.

Autor de correspondencia: Maritza Cárdenas-Calle, Universidad de Guayaquil, Facultad de Ingeniería Química, Ciudadela Universitaria Salvador Allende, Av. Delta y Av. Kennedy Guayaquil, Ecuador.

E-mail: maritza.cardenasc@ug.edu.ec Tlf.Fax: (593-4) 2-286950

\begin{abstract}
Resumen
Se muestrearon siete sitios de buceo en la Reserva Marina El Pelado entre febrero y mayo de 2013, con el objetivo de evaluar la estructura de las comunidades macrobentónicas. La abundancia y diversidad de macroinvertebrados y peces fueron estimadas a lo largo de dos transectos dispuestos paralelos a la costa. Cada transecto fue de $50 \mathrm{~m}$ de longitud, se usaron cuadrantes para cuantificar el porcentaje de cobertura de organismos sésiles. Se midió riqueza, diversidad (Indice Shannon Wiener) y equitabilidad (Índice de Pielou). Se identificaron 64 especies de invertebrados y 50 de peces. Cnidarios, equinodermos, serránidos y pomacéntridos fueron los taxones más abundantes. La mayor diversidad de peces (4 bits.ind $\left.{ }^{-1}\right)$ y macroinvertebrados $\left(2,87\right.$ bits. ind $\left.^{-1}\right)$ estuvo asociada principalmente a fondos rocosos. Los sitios más diversos fueron La Cabeza del Viejo, Zona Protegida, La Pared y El Cuarenta. Sólo se encontraron diferencias significativas $(P$ $<0,05$ ) entre los ensamblajes de macroinvertebrados móviles a nivel de hábitats.
\end{abstract}

\section{Palabras Clave:}

Area protegida, arrecifes coralinos, biodiversidad, macroinvertebrados, turismo marino.

Clasificación JEL: O13, O3.

\begin{abstract}
Seven sites were sampled in seven scuba diving sites in the El Pelado Marine Reserve between February and May 2013, with the objective of evaluating the macrobenthic community structure. The abundance and diversity of macroinvertebrates and fishes were estimated along two transects arranged parallel to the coast. Quadrants were used to quantify the percentage of coverage of sessile organisms. Richness, Diversity (Shannon Wiener Index) and equitability (Pielou Index) were measured. We identified 64 species of invertebrates and 50 species of fish. Cnidarians, echinoderms, serranids and pomacentrids were the most abundant taxa. The greatest diversity of fish (4 bits.ind $\left.{ }^{-1}\right)$ and macroinvertebrates $\left(2.87\right.$ bits.ind $\left.^{-1}\right)$ were associated mainly with rocky bottoms. The most diverse sites were La Cabeza del Viejo, Zona Protegida, La Pared and El Cuarenta. Significant differences $(P<0,05)$ were found between the biothic assemblages of mobile macroinvertebrates by habitats.
\end{abstract}

Keywords:

Biodiversity, coral reef, macroinvertebrates, marine tourism, protected area. JEL Classification: 013, 03. 


\section{Introducción}

Las comunidades macrobentónicas asociadas a los fondos rocosos y arenosos en ecosistemas marino costeros constituyen un eslabón importante al interior de la red trófica; su papel funcional reside en la transferencia de energía entre los productores primarios y consumidores terciarios (Wilbert \& Clarke,1998). Estas comunidades habitan diferentes tipos de sustratos tanto en la zona intermareal y submareal (aguas someras y abisales) (Keen, 1971). Cumplen importantes funciones en el ecosistema como reguladores ecológicos, filtradores (Keen, 1971; Caso, 1994; González-Medina et al., 2006) y bioindicadores de perturbaciones naturales y antrópicas (Glynn et al., 2001).

En Ecuador los estudios de biodiversidad marina reportan hasta el 2003 la presencia de 1859 especies marinas: 479 comerciales y 1380 no comerciales pertenecientes a ocho Phyla siendo los taxones mejores representados los peces y moluscos (Cruz et al., 2003). En la plataforma continental se han identificado 150 especies (120 crustáceos, 15 moluscos, 13 equinodermos $\mathrm{y}$ dos cnidarios) registrados hasta los $200 \mathrm{~m}$, alcanzando una diversidad media $(2,58$; $1,86 ; 2,50$ y 1,26$)$ para los cuatro cruceros de investigación realizados por el $\mathrm{B} / \mathrm{I}$ "Tohalli" del Instituto Nacional de Pesca (Mora et al., 2010). Estudios de la diversidad de las comunidades macrobentónicas a nivel de sustratos rocosos submareales, litorales y pozas intermareales en la costa ecuatoriana son aún incipientes (Cárdenas, 2008; Rivera, 2012; Feijoó, 2013), conociéndose muy poco sobre el efecto de las actividades antrópicas como la sobrepesca (Aguilar et al., 2009; Aguilar et al., 2010), navegación, desarrollo de la infraestructura hotelera, centros poblados, laboratorios, actividades turísticas en la biota marina presente en la Reserva Marina El Pelado.

En esta área protegida, una de las principales actividades recreativas $y$ turísticas que se realiza es el buceo recreativo, actividad que ha tenido un rápido desarrollo por los atractivos $\mathrm{y}$ valores naturales del área, entre los que se encuentran sus bajos rocosos, paredes verticales, zonas arrecifales que son hábitat y refugio de una variedad de peces e invertebrados (Ministerio del Ambiente, 2014). Sus características paisajísticas, biodiversidad, escasas corrientes, buena visibilidad y variados hábitats, propician el desarrollo del buceo; actividad que se realiza en alrededor de 15 bajos, y que ha aumentado gradualmente a partir de la década de los 90 (Cárdenas \& Triviño, 2014), registrándose hasta febrero de 2017 un total de 14014 visitantes según cifras del personal técnico del Ministerio del Ambiente (R. Angel, Comunicación personal), generando oportunidades laborales directas e indirectas a los residentes de la comunidad de Ayangue y comunas aledañas.

Las evaluaciones de las comunidades bentónicas a nivel submareal en la Reserva Marina El Pelado se han enfocado especialmente a estudios poblacionales de recursos pesqueros como la realizada a Isostichopus fuscus (pepino de mar) cuyas densidades registradas en los alrededores 
del Islote El Pelado fueron bajas $(0,01$ ind. $\mathrm{m}^{-2}$ ) (Aguilar et al., 2009); así también el estudio de Spondylus calcifer realizado entre marzo y abril de 2010 en siete sitios, registró sólo un individuo en el bajo La Pared (Aguilar et al., 2010). Entre los primeros inventarios de especies macrobentónicas se encuentran el realizado por Cárdenas en el 2008 en el sitio denominado El Planchón, proporcionando un listado preliminar de 31 especies de macroinvertebrados y 28 especies de peces asociados a fondos rocosos. Posteriormente Rivera en el 2012 amplía los sitios de estudio a tres bajos (La Pared, El Cuarenta y El Pelado Los Corales) y reporta la presencia de 43 especies de invertebrados y 46 de peces, destacándose en ambos estudios la presencia de cnidarios, equinodermos y moluscos.

Estudios relacionados a la composición de peces determinaron la presencia de 11 familias que residen en zonas rocosas, siendo estas: Bleniidae, Cirrhitidae, Chaenopsidae, Chaetodontidae, Gobiidae, Labridae, Labrisomidae, Pomacanthidae, Pomacentridae, Serranidae y Tetraodontidae (Rivera, 2012). Mientras estudios de peces batoideos realizados en el área circundante al Islote El Pelado en el 2013, registraron la presencia de una importante cantidad de individuos (314 rayas, 47 guitarras y 37 torpedos) perteneciente a siete especies, siendo algunas de ellas: Urobatis halleri, Rhinobatos prahli, Narcine entemedor entre otras, y cuyas mayores agregaciones se registraron en los sitios El Arenal y el Acuario (Feijoó, 2013).

Los corales y otros invertebrados sésiles pueden usarse como bioindicadores de la salud ambiental de un ecosistema (Glynn et al., 2001). La actividad turística sin planificación podría generar impactos negativos a la fauna marina que puedan ser detectados por estos bioindicadores. En este estudio se caracterizaron las comunidades de macroinvertebrados, organismos sésiles y peces en los sitios más frecuentados por buzos deportivos en la Reserva Marina El Pelado; con el fin de proporcionar un listado de especies y conocer la abundancia y diversidad de dichas comunidades, lo cual contribuirá con información técnica para el manejo sustentable de los recursos marinos del área y futuras evaluaciones sobre el efecto del buceo recreativo sobre las zonas coralinas y arrecifes rocosos.

\section{Materiales y métodos}

\section{Área de estudio}

La Reserva Marina El Pelado forma parte de las parroquias de Manglaralto y Colonche, en la Provincia de Santa Elena (Figura 1). Tiene una extensión de $13.155,30$ ha $(13.004,75$ ha de la zona marina y 150,55 ha de la zona terrestre incluyendo el perfil costero, manglar y el islote El Pelado). Forma parte del Sistema Nacional de Áreas Protegidas desde el 2012, su área está restringida al desarrollo de actividades de uso múltiple, protección estricta, restauración, uso especial, uso público $\mathrm{y}$ turismo (Ministerio del Ambiente, 2014). Entre los hábitats submareales presentes en esta reserva se encuentran los fondos duros, fondos suaves, fondos mixtos, paredes verticales, arrecifes rocosos, arrecifes 
coralinos (Cárdenas \& Triviño, 2014; Ministerio del Ambiente, 2014) y sustratos artificiales (Ministerio del Ambiente, 2013; Cárdenas \& Triviño, 2014). El borde costero está constituido por la Bahía de Ayangue, playas de barrera, acantilados altos, bajos, un sistema de planicie litoral y flechas de barrera (Boothroyd et al., 1994). Las actividades turísticas que se desarrollan en esta área están relacionadas al disfrute del sol y la playa como otras zonas costeras del Ecuador (Perrone et al., 2009) y además tiene una larga tradición de actividades pesqueras (Ministerio del Ambiente, 2014). Esta reserva es considerada por los turistas como uno de los balnearios favoritos en la costa ecuatoriana, así en el feriado del año nuevo de 2017 se registró la visita de 5350 turistas (http://www.ambiente.gob.ec).

\section{Muestreo}

Los muestreos se realizaron entre febrero y mayo de 2013, a profundidades que oscilaron entre los 8 y 30 metros alrededor del Islote El Pelado (aproximadamente 2,5 millas náuticas de la Bahía de Ayangue). Se seleccionaron siete sitios de muestreo (Figura 1), considerados como los lugares más frecuentados por los buzos según una encuesta realizada a guías, instructores y centros de buceo por Suárez en el 2013. Además, se consideró la representatividad de hábitats (paredes verticales, fondos rocosos, fondos arenosos) y el tipo de sustrato. Los sitios muestreados fueron: El Cuarenta (1 ${ }^{\circ} 56^{\prime} 19,616^{\prime \prime} \mathrm{S}-80^{\circ} 47^{\prime} 11,489^{\prime \prime}$ O); el Barco Hundido (1 ${ }^{\circ} 56^{\prime} 7,240^{\prime \prime} \mathrm{S}$ $80^{\circ} 47^{\prime} 14,857^{\prime \prime}$ O); la Cabeza del Viejo (2³9'36,455" S - 8047'21,499" O). El
Cristo (156'5,742" S - 8047'19,357" O); El Planchón $\left(2^{\circ} 39^{\prime} 28,314 "\right.$ S $80^{\circ} 47^{\prime} 31,929^{\prime \prime} \quad$ O); Zona protegida (1 $\left.{ }^{\circ} 56 ' 5,547^{\prime \prime} \mathrm{S}-80^{\circ} 47^{\prime} 9,292^{\prime \prime} \mathrm{O}\right)$ y La Pared (155'58,057" S - 8047'33,277" O).

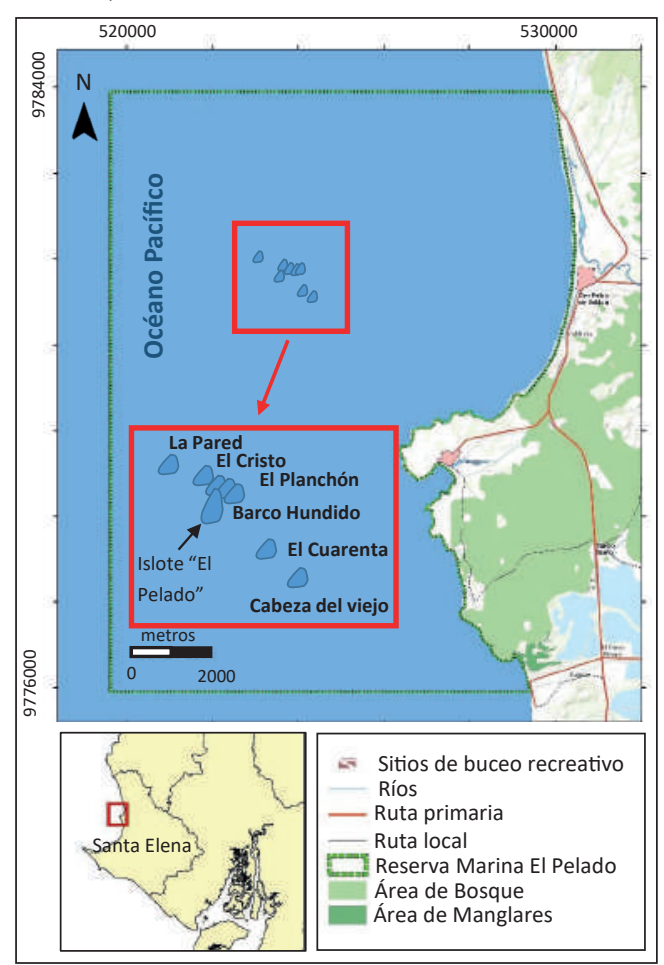

Figura 1. Ubicación geográfica de siete sitios de buceo recreativo situados alrededor del Islote El Pelado en Ayangue durante el primer trimestre de 2013.

Para la cuantificación de macroinvertebrados móviles, organismos sésiles y peces se usaron transectos y cuadrantes. En cada sitio se colocaron dos transectos paralelos a la costa de $50 \mathrm{~m}$ de longitud ubicados entre 8 y $30 \mathrm{~m}$ de profundidad en las distintas localidades (Edgar et al., 2011). Para la evaluación de macroinvertebrados móviles (moluscos, equinodermos y crustáceos $>2,5 \mathrm{~cm}$ de longitud) un buzo se desplazó a lo largo del transecto, contando los organismos presentes a cada lado del transecto en un rango de $1 \mathrm{~m}$ a cada lado del mismo. Se registró el número de individuos de cada 
Comunidades Bentónicas Presentes en Sitios de Buceo en la Reserva Marina El Pelado

especie presente en los dos lados del transecto, muestreando un área total de $100 \mathrm{~m}^{2}$ por transecto. Para los organismos sésiles (invertebrados y macroalgas) se usaron cuadrantes de $1 \mathrm{~m}^{2}$, los mismos que fueron colocados sobre el transecto cada $5 \mathrm{~m}$, se evaluó un total de 10 cuadrantes por cada transecto. Se cuantificaron los puntos de intersección ocupados por esponjas, corales, tunicados, zoantidos y macroalgas, identificados hasta el nivel taxonómico más bajo posible. A partir de estos datos registrados, se determinó la abundancia y diversidad de macroinvertebrados móviles y organismos sésiles. Los censos se realizaron mediante buceo con tanque (SCUBA) y la mayoría de las especies se identificaron in situ, otras que no se lograron identificar en campo fueron transportadas al laboratorio para su clasificación mediante claves taxonómicas específicas. Se utilizaron varias claves taxonómicas para identificar las especies de equinodermos (Caso, 1994; Hickman, 1998), de moluscos (Morris, 1966; Keen, 1971; Hickman \& Finet, 1999), de crustáceos (Hickman \& Todd, 2000), de corales (Hickman, 2008) y de otros invertebrados (Brusca, 1973).

Para estimar la abundancia y diversidad de peces, el buzo se desplazó a lo largo del transecto de $50 \mathrm{~m}$ lineales por una banda de 5 $\mathrm{m}$ a cada lado del transecto, esto permitió cubrir un área de $500 \mathrm{~m}^{2}$ y registrar las especies y el número de individuos de las mismas (Edgar et al., 2011). Adicionalmente, se registraron las especies vistas fuera del transecto durante el censo, éstas fueron incluidas en el registro general de presencia de especies. Para la identificación de peces se usaron guías fotográficas y taxonómicas específicas para la región (Humann, 1993;
Allen \& Robertson, 2006).

\section{Análisis estadísticos}

Se analizó la abundancia de las especies de los grupos estudiados mediante análisis multivariados $\mathrm{y}$ ordenaciones usados en estudios de ecología de comunidades biológicas (Clarke \& Warwick, 2001) con el propósito de comparar las diferencias de los ensamblajes bióticos entre los sitios de estudio, hábitat y tipo de sustrato. Para examinar visualmente los ensamblajes se realizó análisis de escalamiento multidimensional no métrico (nMDS), mediante la ordenación de los datos, estos fueron transformados con raíz cuarta para reducir la influencia de las especies con los valores más altos (Clarke \& Warwick, 1994) y se utilizó el coeficiente de Bray-Curtis como un parámetro de semejanza a través del uso del software Plymouth Routines in Multivariate Ecological Research (PRIMER) versión 6.0. Para estimar la riqueza de especies $(S)$, diversidad $\left(H^{\prime}\right)$ usando logaritmo base dos y la equitabilidad $\left(J^{\prime}\right)$ con el índice de Pielou se usó la rutina DIVERSE del programa PRIMER. Para investigar si existen diferencias en la estructura de la estructura comunitaria a nivel de hábitats, se utilizó un análisis de la similitud (ANOSIM) a una vía, a partir de la matriz de las abundancias luego de comprobar el cumplimiento de normalidad y homogeneidad de varianzas (Zar, 1996).

Para determinar la fragilidad de los sitios, se analizó el estado de conservación de las especies de invertebrados bentónicos con énfasis en corales, basado en la presencia en la Lista Roja de la Unión Internacional para la Conservación de la Naturaleza (IUCN, 2018) 
y en los apéndices del Convenio sobre el Comercio Internacional de Especies Amenazadas de Fauna y Flora Silvestres (CITES, 2018).

\section{Resultados}

Composición y abundancia de la comunidad bentónica

De los sitios muestreados el 31,09\% fueron sustratos arenosos y el 68,91\% fueron rocosos, de estos últimos el $63,48 \%$ estuvieron cubiertos de organismos y el $36,52 \%$ fueron rocas descubiertas. Se identificaron 64 especies (43 sésiles y 21 móviles) contenidas en ocho phyla, 16 clases, 37 familias. Entre las taxa registradas se encontraron: Cnidaria, Porifera, Bryozoa, Echinodermata, Mollusca, Arthropoda, Annelida y Urochordata (Tabla 1). Las especies más Tabla 1 .

Lista de Presencia de Macroinvertebrados Móviles y organismos sésiles $\left(^{*}\right)$ presentes en siete sitios de buceo de la Reserva Marina El Pelado durante 2013. Se incluye la presencia de especies fuera del transecto.

\begin{tabular}{|c|c|c|c|c|c|c|c|c|c|c|c|}
\hline \multirow[b]{2}{*}{ Phylum } & \multirow[b]{2}{*}{ Clase } & \multirow[b]{2}{*}{ Orden } & \multirow[b]{2}{*}{ Familia } & \multirow[b]{2}{*}{ Nombre científico } & \multicolumn{4}{|c|}{ Fondo rocoso } & \multicolumn{3}{|c|}{$\begin{array}{c}\text { Fondo } \\
\text { arenoso }\end{array}$} \\
\hline & & & & & 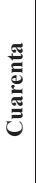 & 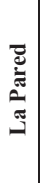 & 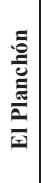 & 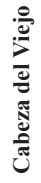 & 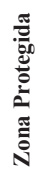 & 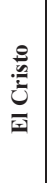 & 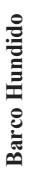 \\
\hline \multirow{25}{*}{ Cnidaria } & \multirow{22}{*}{ Anthozoa } & \multirow{12}{*}{ Alcyonacea } & Gorgoniidae & $\begin{array}{l}\text { *Leptogorgia alba Duchassaing \& Michelotti, } \\
1864\end{array}$ & $\mathrm{x}$ & $\mathrm{x}$ & & $\mathrm{x}$ & & & \\
\hline & & & Gorgoniidae & *Leptogorgia cf. rosada & $\mathrm{x}$ & $\mathrm{x}$ & & $\mathrm{x}$ & & & \\
\hline & & & Gorgoniidae & *Leptogorgia cf. lila & $\mathrm{x}$ & $\mathrm{x}$ & $\mathrm{x}$ & & & & \\
\hline & & & Gorgoniidae & *Leptogorgia spp. Milne Edwards, 1857 & & $\mathrm{x}$ & $\mathrm{x}$ & & $\mathrm{x}$ & & \\
\hline & & & Gorgoniidae & *Pacifigorgia irene Bayer, 1951 & $\mathrm{x}$ & $\mathrm{x}$ & & & $\mathrm{x}$ & & \\
\hline & & & Gorgoniidae & *Pacifigorgia adamsii Verrill, 1868 & $\mathrm{x}$ & & & & & & \\
\hline & & & Clavulariidae & $\begin{array}{l}\text { *Carijoa riisei Duchassaing \& Michelotti, } \\
1860\end{array}$ & $\mathrm{x}$ & & & & & & \\
\hline & & & Plexauridae & ${ }^{*}$ Muricea fruticosa Verrill, 1869 & $x$ & $x$ & $x$ & $x$ & & $x$ & \\
\hline & & & Plexauridae & *Muricea spp. Lamouroux, 1821 & & & $\mathrm{x}$ & $\mathrm{x}$ & & & \\
\hline & & & Plexauridae & *Muricea appressa Verrill, 1864 & $\mathrm{x}$ & $\mathrm{x}$ & $\mathrm{x}$ & $\mathrm{x}$ & $\mathrm{x}$ & $\mathrm{x}$ & \\
\hline & & & Plexauridae & $\begin{array}{l}\text { *Heterogorgia hickmani Breedy \& Guzman, } \\
2005\end{array}$ & $\mathrm{x}$ & $\mathrm{x}$ & & & $\mathrm{x}$ & & \\
\hline & & & Plexauridae & *Heterogorgia spp. Verrill, 1868 & $\mathrm{x}$ & $\mathrm{x}$ & $\mathrm{x}$ & & $\mathrm{x}$ & & \\
\hline & & \multirow{2}{*}{ Antipatharia } & Myriopathidae & *Myriopathes panamensis Verrill, 1869 & $\mathrm{x}$ & $\mathrm{x}$ & & $\mathrm{x}$ & & & \\
\hline & & & Antipathidae & *Antipathes galapagensis Deichmann, 1941 & $\mathrm{x}$ & $\mathrm{x}$ & & & & & \\
\hline & & \multirow{4}{*}{ Scleractinia } & Pocilloporidae & *Pocillopora damicornis Linnaeus, 1758 & $\mathrm{x}$ & & $\mathrm{x}$ & & & & \\
\hline & & & Pocilloporidae & *Pocillopora elegans Dana, 1846 & $\mathrm{x}$ & & & & & & \\
\hline & & & Pocilloporidae & *Pocillopora cf. capitata Verrill, 1864 & & & $\mathrm{x}$ & & $\mathrm{x}$ & & \\
\hline & & & Pocilloporidae & *Pocillopora spp. Lamarck, 1816 & $\mathrm{x}$ & & & & & $\mathrm{x}$ & \\
\hline & & & Dendrophylliidae & *Tubastraea coccinea Lesson, 1829 & $\mathrm{x}$ & $\mathrm{x}$ & $\mathrm{x}$ & $\mathrm{x}$ & $\mathrm{x}$ & $\mathrm{x}$ & \\
\hline & & \multirow{3}{*}{ Zoantharia } & Parazoanthidae & $\begin{array}{l}\text { *Parazoanthus sp. Haddon \& Shackleton, } 1891 \\
\text { (rojo) }\end{array}$ & $\mathrm{x}$ & $\mathrm{x}$ & & & & & \\
\hline & & & Parazoanthidae & *Zoantido ND & & $\mathrm{x}$ & $\mathrm{x}$ & & $\mathrm{x}$ & & \\
\hline & & & Sphenopidae & *Palythoa Lamouroux, 1816 & $\mathrm{x}$ & $\mathrm{x}$ & $\mathrm{x}$ & & & & \\
\hline & \multirow{3}{*}{ Hydrozoa } & \multirow{2}{*}{ Leptothecata } & Aglaopheniidae & *Aglaophenia diegensis Torrey, 1904 & & & $\mathrm{x}$ & & & $\mathrm{x}$ & \\
\hline & & & Aglaopheniidae & *Macrorhynchia philippina Kirchenpauer, 1872 & $\mathrm{x}$ & & $\mathrm{x}$ & & & $\mathrm{x}$ & \\
\hline & & Anthoathecata & Pennariidae & *Pennaria disticha Goldfuss, 1820 & $\mathrm{x}$ & & $\mathrm{x}$ & & & & \\
\hline
\end{tabular}

72

INVESTIGATIO No. 11, noviembre 2018 , Edición Especial, pp. 67-88,

ISSN: 1390 - 6399・ISSN-e: 2602 - 8336 
Comunidades Bentónicas Presentes en Sitios de Buceo en la Reserva Marina El Pelado

\begin{tabular}{|c|c|c|c|c|c|c|c|c|c|c|c|}
\hline \multirow{3}{*}{ Porifera } & \multirow{3}{*}{ Demospongiae } & Dendroceratida & Darwinellidae & *Aplysilla sulfurea Schulze, 1878 & & $\mathrm{x}$ & & & & & \\
\hline & & Verongiida & Aplysinidae & *Aplysina sp. Nardo, 1834 & $\mathrm{x}$ & & $\mathrm{x}$ & & & & \\
\hline & & Poecilosclerida & Tedaniidae & *Tedania sp. Gray, 1867 & $\mathrm{x}$ & $\mathrm{x}$ & & & & & \\
\hline \multirow{5}{*}{ Bryozoa } & \multirow{2}{*}{ Gymnolaemata } & \multirow{2}{*}{ Cheilostomatida } & Bugulidae & *Bugulina californica Robertson, 1905 & $\mathrm{x}$ & & & & & & \\
\hline & & & Bugulidae & *Bugula neritina Linnaeus, 1758 & $\mathrm{x}$ & $\mathrm{x}$ & & $\mathrm{x}$ & $\mathrm{x}$ & $\mathrm{x}$ & \\
\hline & & Cyclostomatida & Crisiidae & *Crisia sp. Lamouroux, 1812 & $\mathrm{x}$ & $\mathrm{x}$ & $\mathrm{x}$ & & $\mathrm{x}$ & & \\
\hline & & & & *Bryozoa lila incrustante & & $\mathrm{x}$ & $\mathrm{x}$ & & $\mathrm{x}$ & & \\
\hline & & Diadematoida & Diadematidae & Astropyga pulvinata Lamarck, 1816 & & $\mathrm{x}$ & & & & & \\
\hline \multirow{12}{*}{ Echinodermata } & \multirow{6}{*}{ Echinoidea } & & Diadematidae & Centrostephanus coronatus Verrill, 1867 & $\mathrm{x}$ & & $\mathrm{x}$ & $\mathrm{x}$ & $\mathrm{x}$ & & \\
\hline & & & Diadematidae & Diadema mexicanum A. Agassiz, 1863 & $\mathrm{x}$ & & $\mathrm{x}$ & $\mathrm{x}$ & $\mathrm{x}$ & & \\
\hline & & Cidaroida & Cidaridae & Eucidaris thouarsii L. Agassiz \& Desor, 1846 & $\mathrm{x}$ & $\mathrm{x}$ & $\mathrm{x}$ & & $\mathrm{x}$ & $\mathrm{x}$ & \\
\hline & & \multirow{3}{*}{ Camarodonta } & Echinometridae & Echinometra vanbrunti A. Agassiz, 1863 & $\mathrm{x}$ & $\mathrm{x}$ & & & $\mathrm{x}$ & $\mathrm{x}$ & \\
\hline & & & Toxopneustidae & Toxopneustes roseus A. Agassiz, 1863 & $\mathrm{x}$ & $\mathrm{x}$ & $\mathrm{x}$ & $\mathrm{x}$ & $\mathrm{x}$ & & \\
\hline & & & Toxopneustidae & Tripneustes depressus A. Agassiz, 1863 & $\mathrm{x}$ & & & & & & \\
\hline & \multirow{3}{*}{ Asteroidea } & \multirow{3}{*}{ Valvatida } & Ophidiasteridae & Pharia pyramidata Gray, 1840 & $\mathrm{x}$ & $\mathrm{x}$ & $\mathrm{x}$ & $\mathrm{x}$ & $\mathrm{x}$ & $\mathrm{x}$ & \\
\hline & & & Ophidiasteridae & Phataria unifascialis Gray, 1840 & $\mathrm{x}$ & $\mathrm{x}$ & $\mathrm{x}$ & $\mathrm{x}$ & $\mathrm{x}$ & & \\
\hline & & & Oreasteridae & Pentaceraster cumingi Gray, 1840 & $\mathrm{x}$ & $\mathrm{x}$ & & & & & $\mathrm{x}$ \\
\hline & \multirow{2}{*}{ Ophiuroidea } & \multirow{2}{*}{ Ophiurida } & Ophiotrichidae & Ophiothela mirabilis Verrill, 1867 & $\mathrm{x}$ & & $\mathrm{x}$ & & & & \\
\hline & & & Ophiocomidae & Ophiocoma aethiops Lütken, 1859 & & & & & $\mathrm{x}$ & & \\
\hline & Holothuroidea & Synallactida & Stichopodidae & Isostichopus fuscus Ludwig, 1875 & $\mathrm{x}$ & $\mathrm{x}$ & & & $\mathrm{x}$ & & \\
\hline \multirow{9}{*}{ Mollusca } & \multirow{5}{*}{ Gastropoda } & \multirow{5}{*}{ Neogastropoda } & Muricidae & Hexaplex princeps Broderip, 1833 & $\mathrm{x}$ & & $\mathrm{x}$ & & & & \\
\hline & & & Muricidae & Hexaplex spp. Perry, 1810 & & $\mathrm{x}$ & & & $\mathrm{x}$ & & \\
\hline & & & Fasciolariidae & Triplofusus princeps G. B. Sowerby I, 1825 & $\mathrm{x}$ & & & & & $\mathrm{x}$ & \\
\hline & & & & Conus sp. Linnaeus, 1758 & $\mathrm{x}$ & & $\mathrm{x}$ & & & & \\
\hline & & & Plakobranchidae & Elysia diomedea Bergh, 1894 & $\mathrm{x}$ & & & & & & \\
\hline & \multirow{3}{*}{ Bivalvia } & \multirow{2}{*}{ Ostreida } & Pteriidae & *Pinctada mazatlanica Hanley, 1856 & $\mathrm{x}$ & $\mathrm{x}$ & & & & & \\
\hline & & & Pteriidae & *Pteria sterna Gould, 1851 & $\mathrm{x}$ & & & & & & \\
\hline & & Pectinida & Spondylidae & *Spondylus calcifer Carpenter, 1857 & $\mathrm{x}$ & & & & & & \\
\hline & Cephalopoda & Octopoda & Octopodidae & Octopus sp. Cuvier, 1798 & $\mathrm{x}$ & $\mathrm{x}$ & & & $\mathrm{x}$ & & \\
\hline \multirow{2}{*}{ Arthropoda } & Malacostraca & Decapoda & Trapeziidae & Trapezia ferruginea Latreille, 1828 & $\mathrm{x}$ & & & & & & \\
\hline & Hexanauplia & Sessilia & Balanidae & *Megabalanus peninsularis Pilsbry, 1916 & $\mathrm{x}$ & & $\mathrm{x}$ & & $\mathrm{x}$ & & \\
\hline \multirow{3}{*}{ Annelida } & \multirow{3}{*}{ Polychaeta } & Sabellida & Serpulidae & *Spirobranchus giganteus Pallas, 1766 & $\mathrm{x}$ & $\mathrm{x}$ & $\mathrm{x}$ & & $\mathrm{x}$ & & \\
\hline & & & Serpulidae & *Serpulidae Rafinesque, 1815 & & & & & & $\mathrm{x}$ & \\
\hline & & & & *Poliqueto ND & & & & & & $\mathrm{x}$ & \\
\hline & & & Didemnidae & *Didemnum sp1 Savigny, 1816 blanco & $\mathrm{x}$ & & $\mathrm{x}$ & & $\mathrm{x}$ & & \\
\hline & & & Didemnidae & *Didemnum cf. cineraceum Sluiter, 1898 & & & $\mathrm{x}$ & & & & \\
\hline & & Aplousobranchia & Didemnidae & *Didemnum perlucidum Monniot F., 1983 & $\mathrm{x}$ & & & & & & \\
\hline Chordata & Ascidiacea & & Polycitoridae & *Eudistoma sp. Caullery, 1909 & $\mathrm{x}$ & & $\mathrm{x}$ & & & & \\
\hline & & & Polycitoridae & ${ }^{*}$ Cystodytes sp. Drasche, 1884 & $\mathrm{x}$ & & & & & & \\
\hline & & Phlebobranchia & Ascidiidae & *Ascidia sp 1 Linnaeus, 1767 (café) & $\mathrm{x}$ & & & $\mathrm{x}$ & & & \\
\hline & & & Ascidiidae & *Ascidia sp 2 Linnaeus, 1767 (blanca) & & & & $\mathrm{x}$ & & & \\
\hline & Thaliacea & Salpida & Salpidae & Salpa sp. Forskål, 1775 & & & $\mathrm{x}$ & & & & \\
\hline Chlorophyta & & & & *Chlorophyta & & & & & & & \\
\hline Ochrophyta & Phaeophyceae & Dictyotales & Dictyotaceae & *Padina sp. Adanson, 1763 & & & & & & & \\
\hline Complejos & Complejo Bug & ula neritina, Litho & thamnium y $M e g$ & abalanus peninsularis & $\mathrm{x}$ & $\mathrm{x}$ & & & & & \\
\hline bióticos & Bugula neritin & $a$ con rodofitas fil & amentosas & & & & & & $\mathrm{x}$ & & \\
\hline
\end{tabular}

\section{3}

INVESTIGATIO No. 11, noviembre 2018,

Edición Especial, pp. 67-88,

ISSN: 1390 - 6399・ISSN-e: 2602 - 8336 
abundantes a nivel de macroinvertebrados móviles fueron Phataria unifascialis, Echinometra vanbrunti y Centrostephanus coronatus, las cuales juntas constituyen aproximadamente el $63 \%$ del total de individuos contados. P. unifascialis y $E$. vanbrunti alcanzaron sus mayores porcentajes en la Pared (estructura rocosa vertical) y $C$. coronatus en la Cabeza del Viejo (zona rocosa) (Tabla 2). Los organismos sésiles más representativos fueron los Cnidarios representados especialmente por los octocorales (Muricea appressa, Muricea fruticosa, Heterogorgia hickmani, Leptogorgia alba, Leptogorgia cf. rosada), el coral ahermatípico Tubastraea coccinea, los corales córneos: Antipathes galapagensis y Myriopathes panamensis, corales escleractínidos: Pocillopora damicornis, Pocillopora spp., P. elegans, P. cf. capitata. Otras especies abundantes fueron Bugula neritina, Lithothamnium, Megabalanus peninsularis y rodofitas filamentosas.

Los sitios de buceo con mayor cobertura de sésiles fueron La Cabeza del Viejo y El Cuarenta, del total de organismos sésiles contados, el 12,28\% correspondió a $M$. panamensis, especie que se registró en los sitios con mayores profundidades como El Cuarenta, La Cabeza del Viejo y La Pared localizados entre 12 a $17 \mathrm{~m}$. En menor porcentaje se registraron los corales Pocillopora (Figura 2) con el 3\%. De los

Tabla 2

Abundancia relativa de macroinvertebrados móviles registrados en los transectos de los siete sitios de buceo del Islote El Pelado en el 2013.

\begin{tabular}{|c|c|c|c|c|c|c|c|c|}
\hline Taxa & Especies & Cuarenta & $\begin{array}{c}\text { Barco } \\
\text { Hundido }\end{array}$ & $\begin{array}{c}\text { El } \\
\text { Planchón }\end{array}$ & $\begin{array}{c}\text { El } \\
\text { Cristo }\end{array}$ & $\begin{array}{c}\text { Zona } \\
\text { Protegida } \\
\end{array}$ & $\begin{array}{c}\text { La } \\
\text { Pared }\end{array}$ & $\begin{array}{c}\text { Cabeza del } \\
\text { Viejo }\end{array}$ \\
\hline \multirow{11}{*}{ Equinodermos } & $\begin{array}{l}\text { Centrostephanus } \\
\text { coronatus }\end{array}$ & 60 & 0 & 1 & 0 & 13 & 0 & 136 \\
\hline & Diadema mexicanum & 17 & 0 & 101 & 0 & 6 & 0 & 3 \\
\hline & Eucidaris thouarsii & 2 & 0 & 0 & 6 & 35 & 63 & 0 \\
\hline & $\begin{array}{l}\text { Echinometra } \\
\text { vanbrunti }\end{array}$ & 0 & 0 & 0 & 19 & 43 & 161 & 0 \\
\hline & Toxopneustes roseus & 0 & 0 & 0 & 0 & 10 & 0 & 2 \\
\hline & Tripneustes depressus & 1 & 0 & 0 & 0 & 0 & 0 & 0 \\
\hline & Pharia pyramidata & 7 & 0 & 2 & 0 & 20 & 19 & 36 \\
\hline & Phataria unifascialis & 65 & 0 & 7 & 6 & 25 & 87 & 56 \\
\hline & Pentaceraster cumingi & 0 & 9 & 0 & 0 & 0 & 1 & 0 \\
\hline & Ophiocoma aethiops & 0 & 0 & 0 & 0 & 4 & 0 & 0 \\
\hline & Isostichopus fuscus & 0 & 0 & 0 & 0 & 2 & 16 & 0 \\
\hline \multirow{7}{*}{ Moluscos } & Hexaplex princeps & 0 & 0 & 2 & 0 & 0 & 7 & 0 \\
\hline & Hexaplex spp. & 0 & 0 & 0 & 0 & 4 & 0 & 0 \\
\hline & Triplofusus princeps & 0 & 0 & 0 & 2 & 0 & 0 & 0 \\
\hline & Conus sp. & 2 & 0 & 1 & 0 & 0 & 0 & 0 \\
\hline & Octopus sp. & 0 & 0 & 0 & 0 & 1 & 12 & 0 \\
\hline & Total especies & 7 & 1 & 6 & 4 & 11 & 8 & 5 \\
\hline & Total individuos & 161 & 10 & 120 & 37 & 174 & 374 & 238 \\
\hline
\end{tabular}



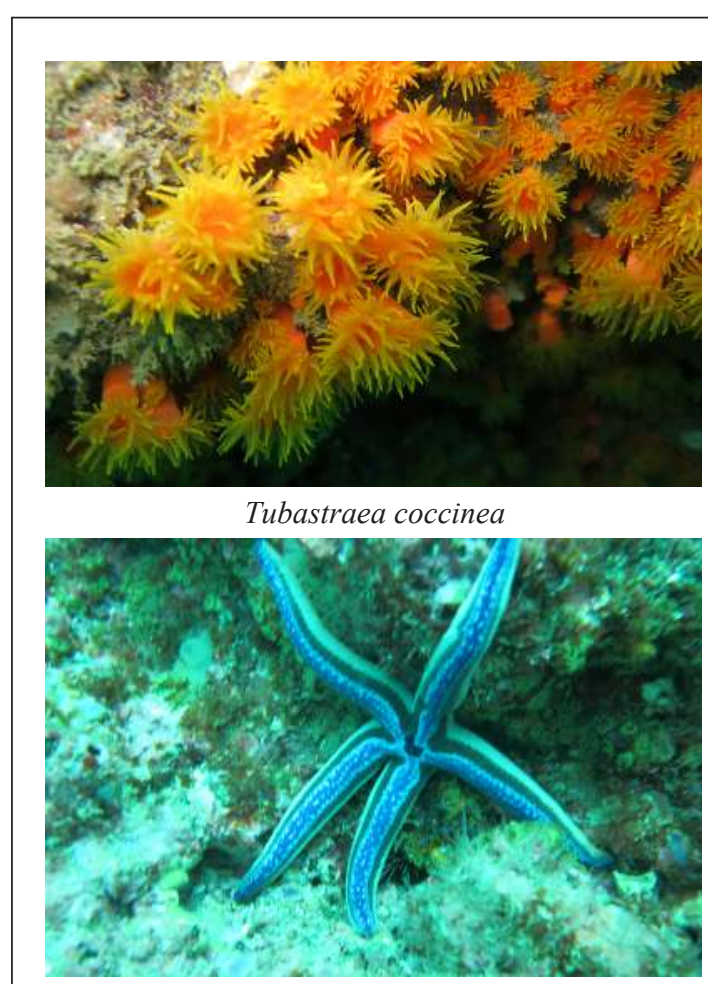

Phataria unifascialis

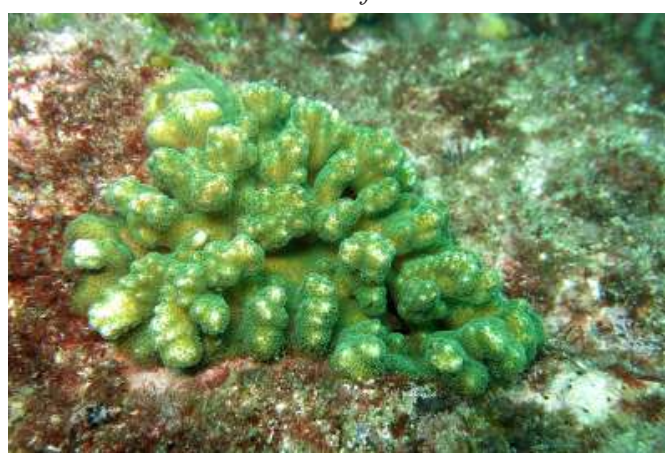

Pocillopora spp.

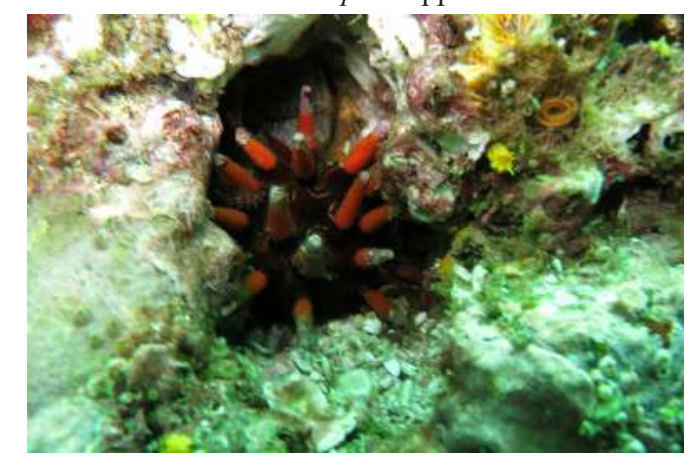

Eucidaris thouarsii
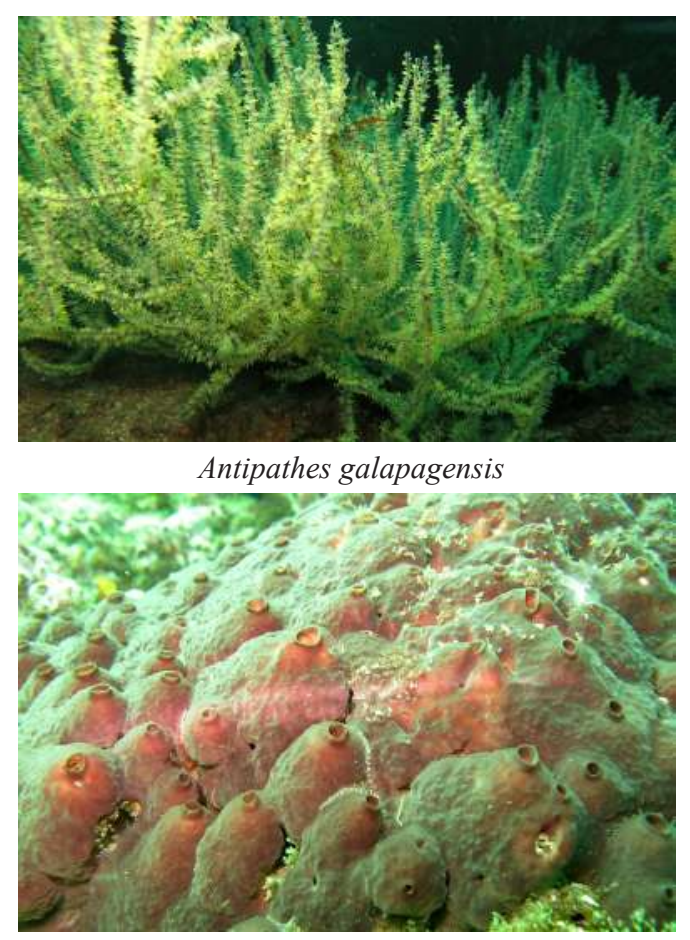

Aplysina sp.

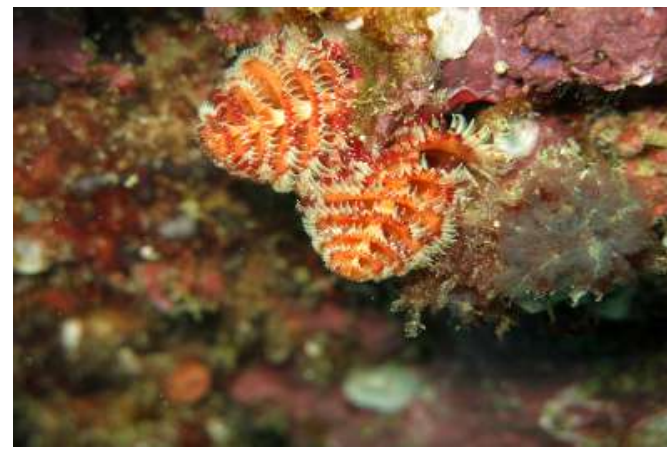

Spirobranchus giganteus

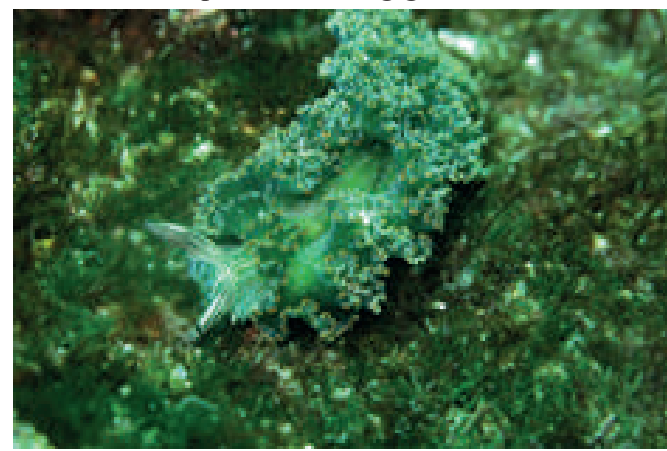

Elysia diomedea

Figura 2. Principales especies de invertebrados registrados en los sitios de buceo situados alrededor del Islote El Pelado en la Reserva Marina El Pelado. 
corales registrados Pocillopora damicornis y $P$. capitata, se encuentran listados en IUCN como especies de preocupación menor y $P$. elegans como una especie vulnerable. Así como Isostichopus fuscus está considerada como una especie en peligro de extinción (IUCN, 2018). Mientras que los corales negros Antipathes galapagensis y Myriopathes panamesis forman parte del Apéndice II de CITES como otros Antipatharios (CITES, 2018). Así también Isostichopus fuscus está considerada como una especie en peligro de extinción (IUCN, 2018).

\section{Afinidad entre localidades}

El gráfico de escalamiento multidimensional mostró que el ensamblaje de macroinvertebrados móviles presenta cierta similitud entre la Cabeza del Viejo y el Cuarenta; así como entre La Zona Protegida y La Pared (Figura 3A). El análisis de similaridad ANOSIM dio un nivel de significancia de $1.9 \%$ para los diferentes

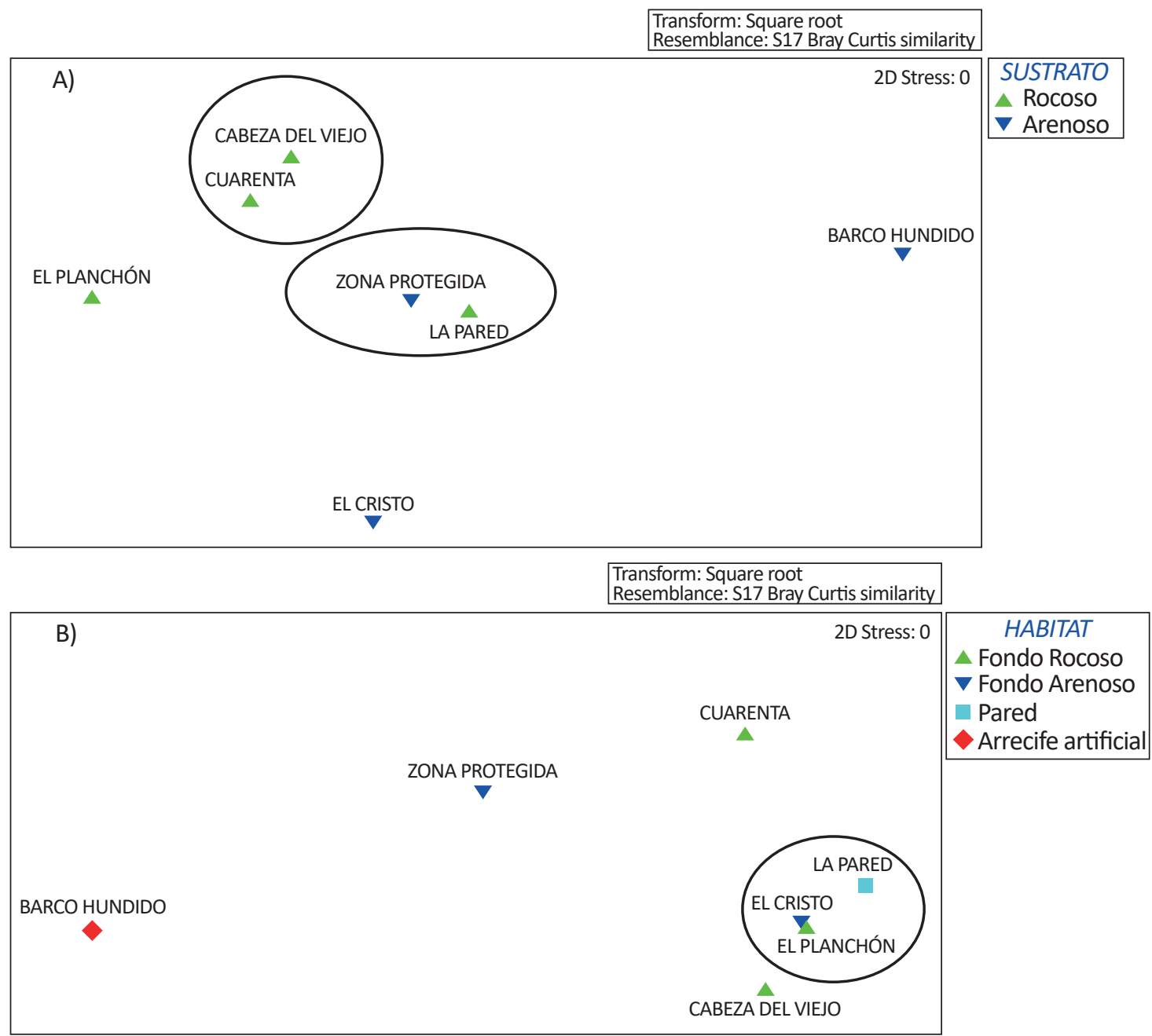

Figura 3. Escalamiento multidimensional no métrico basado en el índice de similitud de Bray-Curtis de la composición y abundancia del ensamblaje de macroinvertebrados móviles según el sustrato A) y organismos sésiles B) según los hábitats registrados en los sitios de muestreo en la Reserva Marina El Pelado. 
hábitats, con un $R$ global de 0.79 por lo que se rechaza la hipótesis nula, indicando que existen diferencias entre los macroinvertebrados en relación al hábitat (fondos arenosos, rocosos, pared y arrecife artificial). La similitud entre organismos sésiles a nivel de hábitat fue mayor entre El Planchón, La Pared y El Cristo (zona arenosa con parches rocosos) (Figura 3B). Sin embargo, el ensamblaje de organismos sésiles no mostró diferencias significativas entre las estaciones (significancia 12,4\% y $R$ de 0.412).

Las fluctuaciones espaciales de la diversidad de las comunidades bentónicas se muestran en la Tabla 3. Los índices de diversidad y equitabilidad fueron mayores a nivel de invertebrados móviles en comparación con los sésiles, registrándose la mayor diversidad en fondos rocosos $\left(H^{\prime}=1,55 \quad\right.$ bits.ind $^{-1} \quad$ y $\quad J=0,57 \quad$ en macroinvertebrados) y en organismos sésiles $\left(H^{\prime}=1,79\right.$ bits.ind $^{-1}$ y $\left.J=0,52\right)$. Los sitios más diversos a nivel de macroinvertebrados móviles fueron la zona protegida, La Pared, y El Cuarenta $\left(H^{\prime}=2,87-1,82\right.$ bits.ind $\left.^{-1}\right)$ y a nivel de organismos sésiles La Pared, El Cristo y El Planchón $\left(H^{\prime}=1,99-1,77\right.$ bits.ind $\left.^{-1}\right)$ (Tabla 3).

\section{Composición y abundancia de peces}

Se identificó un total de 50 especies, contenidas en 23 familias (Tabla 4), siendo las más representativas: Serranidae y Pomacentridae. El mayor número de especies correspondieron a: Serranidae (siete), seguida de Pomacentridae (seis), Haemulidae (cinco). Las especies más abundantes que alcanzaron entre 26 a 40 individuos fueron Paranthias colonus, Halichoeres dispilus y Johnrandallia

Tabla 3.

Índices de Diversidad (Índice Shannon Wiener $\left(H^{\prime}\right)$ e índice de Simpson $(D)$ y Equitabilidad: Índice de Pielou $(J)$ de invertebrados móviles y organismos sésiles presentes en los sitios de estudio. La zona protegida presenta un sustrato predominantemente arenoso con parches rocosos y coralinos $(*)$.

\begin{tabular}{|c|c|c|c|c|c|}
\hline Sustrato & Sitios & $\begin{array}{l}\text { Número de } \\
\text { Especies }(S)\end{array}$ & $\begin{array}{c}\text { Índice Shannon } \\
\text { Wiener }\left(H^{\prime}\right)\end{array}$ & $\begin{array}{c}\text { Índice de } \\
\text { Simpson }(D)\end{array}$ & $\begin{array}{c}\text { Equitabilidad } \\
(J)\end{array}$ \\
\hline \multicolumn{6}{|c|}{ MACRO INVERTEBRADOS MÓVILES } \\
\hline \multirow[t]{4}{*}{ Fondo Rocoso } & La Pared & 8 & 2,16 & 0,72 & 0,72 \\
\hline & El Cuarenta & 7 & 1,82 & 0,66 & 0,64 \\
\hline & Cabeza del Viejo & 5 & 1,5 & 0,58 & 0,65 \\
\hline & El Planchón & 6 & 0,73 & 0,21 & 0,28 \\
\hline \multirow[t]{3}{*}{ Fondo arenoso } & Zona Protegida & 11 & 2,87 & 0,84 & 0,83 \\
\hline & El Cristo & 4 & 1,59 & 0,62 & 0,79 \\
\hline & El Barco Hundido & 1 & 0 & 0 & 0 \\
\hline \multicolumn{6}{|c|}{$\begin{array}{c}\text { ORGANISMOS SÉSILES } \\
\end{array}$} \\
\hline \multirow[t]{4}{*}{ Fondo Rocoso } & La Pared & 8 & 1,99 & 0,65 & 0,66 \\
\hline & El Planchón & 15 & 1,77 & 0,67 & 0,45 \\
\hline & El Cuarenta & 14 & 1,75 & 0,63 & 0,46 \\
\hline & Cabeza del Viejo & 10 & 1,63 & 0,58 & 0,49 \\
\hline \multirow[t]{3}{*}{ Fondo arenoso } & El Cristo & 12 & 1,95 & 0,7 & 0,54 \\
\hline & *Zona Protegida & 6 & 0,81 & 0,65 & 0,31 \\
\hline & El Barco Hundido & 0 & 0 & 0 & 0 \\
\hline
\end{tabular}


nigrirostris. Otras especies representativas del área fueron: Stegastes sp., Prionurus laticlavius, Chaetodon humeralis, Anisostremus taeniathus y Abudefduf

Tabla 4.

Lista de especies de peces presentes en los sitios de muestreo.

\begin{tabular}{|c|c|c|c|c|c|c|c|c|}
\hline \multirow{2}{*}{ Familia } & \multirow{2}{*}{ Especies } & \multicolumn{4}{|c|}{ Fondo rocoso } & \multicolumn{3}{|c|}{ Fondo arenoso } \\
\hline & & $\begin{array}{l}\text { El } \\
\text { Cuarenta }\end{array}$ & $\begin{array}{l}\text { La } \\
\text { Pared }\end{array}$ & $\begin{array}{l}\text { El } \\
\text { Planchón }\end{array}$ & $\begin{array}{l}\text { Cabeza del } \\
\text { Viejo }\end{array}$ & $\begin{array}{l}\text { Zona } \\
\text { Protegida }\end{array}$ & $\begin{array}{l}\text { El } \\
\text { Cristo }\end{array}$ & $\begin{array}{l}\text { Barco } \\
\text { Hundido }\end{array}$ \\
\hline Pomacentridae & $\begin{array}{l}\text { Abudefduf } \\
\text { troschelii Gill, } \\
1862\end{array}$ & 27 & 3 & 0 & 8 & 2 & 14 & 8 \\
\hline Monacanthidae & $\begin{array}{l}\text { Aluterus scriptus } \\
\text { Osbeck, } 1765\end{array}$ & 1 & 3 & 1 & 1 & 0 & 0 & 3 \\
\hline Serranidae & $\begin{array}{l}\text { Alphestes } \\
\text { immaculatus } \\
\text { Breder, } 1936\end{array}$ & 0 & 1 & 0 & 0 & 0 & 0 & 0 \\
\hline Aulostomidae & $\begin{array}{l}\text { Aulostomus } \\
\text { chinensis } \\
\text { Linnaeus, } 1766\end{array}$ & 0 & 0 & 0 & 1 & 0 & 0 & 0 \\
\hline Haemulidae & $\begin{array}{l}\text { Anisostremus sp. } \\
\text { Gill, } 1861\end{array}$ & 0 & 0 & 0 & 0 & 0 & 0 & 1 \\
\hline Haemulidae & $\begin{array}{l}\text { Anisotremus } \\
\text { taeniatus Gill, } \\
1861\end{array}$ & 0 & 15 & 0 & 0 & 0 & 0 & 0 \\
\hline Haemulidae & $\begin{array}{l}\text { Anisostremus } \\
\text { interruptus Gill, } \\
1862\end{array}$ & 0 & 5 & 0 & 0 & 0 & 0 & 0 \\
\hline Labridae & $\begin{array}{l}\text { Bodianus } \\
\text { diplotaenia Gill, } \\
1862\end{array}$ & 0 & 0 & 2 & 13 & 0 & 0 & 2 \\
\hline Sparidae & $\begin{array}{l}\text { Calamus } \\
\text { sp. Swainson, } \\
1839\end{array}$ & 0 & 0 & 0 & 3 & 0 & 0 & 0 \\
\hline Tetraodontidae & $\begin{array}{l}\text { Canthigaster } \\
\text { janthinoptera } \\
\text { Bleeker, } 1855\end{array}$ & 0 & 0 & 0 & 1 & 0 & 0 & 0 \\
\hline Tetraodontidae & $\begin{array}{l}\text { Canthigaster sp. } \\
\text { Swainson, } 1839\end{array}$ & 0 & 0 & 0 & 0 & 0 & 1 & 1 \\
\hline Chaetodontidae & $\begin{array}{l}\text { Chaetodon } \\
\text { humeralis } \\
\text { Günther, } 1860\end{array}$ & 7 & 5 & 26 & 0 & 2 & 3 & 31 \\
\hline Pomacentridae & $\begin{array}{l}\text { Chromis } \\
\text { atrilobata } \text { Gill, } \\
1862\end{array}$ & 0 & 0 & 0 & 1 & 0 & 0 & 0 \\
\hline Gobiidae & $\begin{array}{l}\text { Coryphopterus } \\
\text { urospilus } \\
\text { Ginsburg, } 1938\end{array}$ & 0 & 0 & 0 & 1 & 0 & 0 & 0 \\
\hline Serranidae & $\begin{array}{l}\text { Cratinus } \\
\text { agassizii } \\
\text { Steindachner, } \\
1878\end{array}$ & 1 & 0 & 0 & 1 & 0 & 0 & 2 \\
\hline Serranidae & $\begin{array}{l}\text { Cratinus sp. } \\
\text { Steindachner, } \\
1878\end{array}$ & 0 & 0 & 0 & 0 & 0 & 0 & 1 \\
\hline Diodontidae & $\begin{array}{l}\text { Cyclichthys } \\
\text { spilostylus Leis } \\
\text { \& Randall, } 1982\end{array}$ & 0 & 0 & 0 & 0 & 0 & 0 & 5 \\
\hline Diodontidae & $\begin{array}{l}\text { Diodon } \\
\text { holocanthus } \\
\text { Linnaeus, } 1758\end{array}$ & 1 & 12 & 0 & 1 & 1 & 0 & 1 \\
\hline
\end{tabular}

\section{8}

INVESTIGATIO No. 11, noviembre 2018,

Edición Especial, pp. 67-88,

ISSN: 1390 - 6399・ISSN-e: 2602 - 8336 troschelii. Las especies más frecuentes en los conteos fueron Abudefduf troschelii, Chaetodon humeralis y Johnrandallia nigrirostris quienes estuvieron presentes 
Comunidades Bentónicas Presentes en Sitios de Buceo en la Reserva Marina El Pelado

\begin{tabular}{|c|c|c|c|c|c|c|c|c|}
\hline \multirow{2}{*}{ Familia } & \multirow{2}{*}{ Especies } & \multicolumn{4}{|c|}{ Fondo rocoso } & \multicolumn{3}{|c|}{ Fondo arenoso } \\
\hline & & $\begin{array}{l}\text { El } \\
\text { Cuarenta }\end{array}$ & $\begin{array}{l}\text { La } \\
\text { Pared }\end{array}$ & $\begin{array}{l}\text { El } \\
\text { Planchón }\end{array}$ & $\begin{array}{l}\text { Cabeza del } \\
\text { Viejo }\end{array}$ & $\begin{array}{l}\text { Zona } \\
\text { Protegida }\end{array}$ & $\begin{array}{l}\text { El } \\
\text { Cristo }\end{array}$ & $\begin{array}{l}\text { Barco } \\
\text { Hundido }\end{array}$ \\
\hline Gobiidae & $\begin{array}{l}\text { Elacantinus } \\
\text { punticulatus } \\
\text { Ginsburg, } 1938\end{array}$ & 2 & 1 & 0 & 3 & 0 & 0 & 1 \\
\hline Serranidae & $\begin{array}{l}\text { Epinephelus } \\
\text { labriformis } \\
\text { Jenyns, } 1840\end{array}$ & 1 & 3 & 0 & 1 & 0 & 1 & 0 \\
\hline Fistulariidae & $\begin{array}{l}\text { Fistularia } \\
\text { commersonii } \\
\text { Rüppell, } 1838\end{array}$ & 3 & 0 & 0 & 0 & 0 & 0 & 0 \\
\hline Haemulidae & $\begin{array}{l}\text { Haemulon } \\
\text { scudderii Gill, } \\
1862\end{array}$ & 0 & 0 & 0 & 0 & 0 & 0 & 2 \\
\hline Haemulidae & $\begin{array}{l}\text { Haemulon } \\
\text { Cuvier, } 1829\end{array}$ & 0 & 0 & 0 & 0 & 0 & 0 & 1 \\
\hline Labridae & $\begin{array}{l}\text { Halichoeres } \\
\text { dispilus } \\
\text { Günther, } 1864\end{array}$ & 7 & 16 & 5 & 24 & 0 & 0 & 91 \\
\hline Labridae & $\begin{array}{l}\text { Halichoeres } \\
\text { nicholsi Jordan } \\
\text { \& Gilbert, } 1882\end{array}$ & 1 & 0 & 0 & 1 & 0 & 0 & 2 \\
\hline Pomacanthidae & $\begin{array}{l}\text { Holacanthus } \\
\text { passer } \\
\text { Valenciennes, } \\
1846\end{array}$ & 2 & 0 & 0 & 8 & 0 & 0 & 3 \\
\hline Chaetodontidae & $\begin{array}{l}\text { Johnrandallia } \\
\text { nigrirostris Gill, } \\
1862\end{array}$ & 13 & 9 & 35 & 19 & 0 & 69 & 8 \\
\hline Lutjanidae & $\begin{array}{l}\text { Lutjanus Bloch, } \\
1790\end{array}$ & 0 & 0 & 0 & 1 & 0 & 0 & 0 \\
\hline Lutjanidae & $\begin{array}{l}\text { Lutjanus } \\
\text { argentiventris } \\
\text { Peters, } 1869\end{array}$ & 0 & 0 & 0 & 0 & 5 & 0 & 0 \\
\hline Myliobatidae & $\begin{array}{l}\text { Mobula birostris } \\
\text { Walbaum, } 1792\end{array}$ & 0 & 3 & 0 & 0 & 0 & 0 & 0 \\
\hline Muraenidae & Muraena sp. & 0 & 0 & 0 & 0 & 1 & 0 & 0 \\
\hline Blenniidae & $\begin{array}{l}\text { Ophioblennius } \\
\text { steindachneri } \\
\text { Jordan \& } \\
\text { Evermann, } 1898\end{array}$ & 1 & 4 & 2 & 1 & 0 & 1 & 1 \\
\hline Serranidae & $\begin{array}{l}\text { Paranthias } \\
\text { colonus } \\
\text { Valenciennes, } \\
1846\end{array}$ & 156 & 28 & 1 & 9 & 0 & 0 & 8 \\
\hline Pomacanthidae & $\begin{array}{l}\text { Pomacanthus } \\
\text { zonipectus Gill, } \\
1862\end{array}$ & 2 & 17 & 1 & 3 & 0 & 0 & 4 \\
\hline Acanthuridae & $\begin{array}{l}\text { Prionurus } \\
\text { laticlavius } \\
\text { Valenciennes, } \\
1846\end{array}$ & 0 & 6 & 1 & 46 & 0 & 0 & 11 \\
\hline Balistidae & $\begin{array}{l}\text { Pseudobalistes } \\
\text { naufragium } \\
\text { Jordan \& Starks, } \\
1895\end{array}$ & 0 & 0 & 0 & 1 & 0 & 0 & 0 \\
\hline Rhinobatidae & $\begin{array}{l}\text { Pseudobatos } \\
\text { prahli Acero P. } \\
\text { \& Franke, } 1995\end{array}$ & 0 & 0 & 0 & 0 & 1 & 0 & 0 \\
\hline Serranidae & $\begin{array}{l}\text { Rypticus bicolor } \\
\text { Valenciennes, } \\
1846\end{array}$ & 1 & 2 & 0 & 1 & 0 & 0 & 0 \\
\hline
\end{tabular}




\begin{tabular}{|c|c|c|c|c|c|c|c|c|}
\hline \multirow{2}{*}{ Familia } & \multirow{2}{*}{ Especies } & \multicolumn{4}{|c|}{ Fondo rocoso } & \multicolumn{3}{|c|}{ Fondo arenoso } \\
\hline & & $\begin{array}{l}\text { El } \\
\text { Cuarenta }\end{array}$ & $\begin{array}{l}\mathrm{La} \\
\text { Pared }\end{array}$ & $\begin{array}{l}\text { El } \\
\text { Planchón }\end{array}$ & $\begin{array}{l}\text { Cabeza del } \\
\text { Viejo }\end{array}$ & $\begin{array}{l}\text { Zona } \\
\text { Protegida }\end{array}$ & $\begin{array}{l}\text { El } \\
\text { Cristo }\end{array}$ & $\begin{array}{l}\text { Barco } \\
\text { Hundido }\end{array}$ \\
\hline Scaridae & $\begin{array}{l}\text { Scarus perrico } \\
\text { Jordan \& Gilbert, } \\
1882\end{array}$ & 12 & 12 & 1 & 0 & 0 & 0 & 2 \\
\hline Scaridae & $\begin{array}{l}\text { Scarus Forsskål, } \\
1775\end{array}$ & 0 & 0 & 0 & 0 & 0 & 1 & 0 \\
\hline Serranidae & $\begin{array}{l}\text { Serranus } \\
\text { psittacinus } \\
\text { Valenciennes, } \\
1846\end{array}$ & 2 & 2 & 0 & 0 & 0 & 0 & 0 \\
\hline Scorpaenidae & $\begin{array}{l}\text { Scorpaena mystes } \\
\text { Jordan \& Starks, } \\
1895\end{array}$ & 0 & 0 & 0 & 1 & 0 & 0 & 0 \\
\hline Scorpaenidae & $\begin{array}{l}\text { Scorpaena } \\
\text { Linnaeus, } 1758\end{array}$ & 1 & 0 & 0 & 0 & 0 & 0 & 0 \\
\hline Pomacentridae & $\begin{array}{l}\text { Stegastes } \\
\text { acapulcoensis } \\
\text { Fowler, } 1944\end{array}$ & 2 & 14 & 0 & 2 & 0 & 0 & 0 \\
\hline Pomacentridae & $\begin{array}{l}\text { Stegastes beebei } \\
\text { Nichols, } 1924\end{array}$ & 0 & 1 & 5 & 0 & 0 & 0 & 1 \\
\hline Pomacentridae & $\begin{array}{l}\text { Stegastes } \\
\text { flavilatus Gill, } \\
1862\end{array}$ & 0 & 1 & 0 & 0 & 0 & 0 & 0 \\
\hline Pomacentridae & $\begin{array}{l}\text { Stegastes Jenyns, } \\
1840\end{array}$ & 17 & 0 & 0 & 0 & 0 & 0 & 0 \\
\hline Tetraodontidae & $\begin{array}{l}\text { Sphoeroides } \\
\text { lobatus } \\
\text { Steindachner, } \\
1870\end{array}$ & 0 & 0 & 0 & 0 & 2 & 0 & 0 \\
\hline Labridae & $\begin{array}{l}\text { Thalassoma } \\
\text { lucasanum Gill, } \\
1862\end{array}$ & 21 & 5 & 25 & 6 & 0 & 2 & 2 \\
\hline \multirow[t]{3}{*}{ Urotrygonidae } & $\begin{array}{l}\text { Urolophus halleri } \\
\text { Cooper, } 1863\end{array}$ & 0 & 3 & 0 & 2 & 1 & 0 & 1 \\
\hline & Total especies & 22 & 24 & 12 & 27 & 8 & 8 & 25 \\
\hline & Total individuos & 303 & 195 & 117 & 187 & 23 & 100 & 218 \\
\hline
\end{tabular}

en seis de los siete sitios estudiados (Tabla 4). La mayor abundancia de peces se registró en los bajos El Cuarenta y Barco Hundido.

\section{Afinidad entre localidades}

El nMDS mostró que la estructura comunitaria de peces fue más similar entre la Cabeza del Viejo y El Planchón, así como entre el Cuarenta y el Barco Hundido, diferenciándose de los ensamblajes bióticos de la Pared y El Cristo (Figura 4). Sin embargo, no se encontraron diferencias significativas en la estructura comunitaria de peces a nivel de hábitats debido a que el análisis de ANOSIM presentó un nivel de significancia de $43,3 \%$ con un $R$ global de 0,147 .

Los valores de diversidad fueron mayores en fondos rocosos $\left(H^{\prime}=4-2,48\right.$ bits.ind $\left.{ }^{-1}\right)$ así como de equidad $(J=0,57-$ $0,88)$. Los sitios más diversos fueron la Pared, la Cabeza del Viejo, el Barco Hundido y El Cuarenta y el menos diverso 


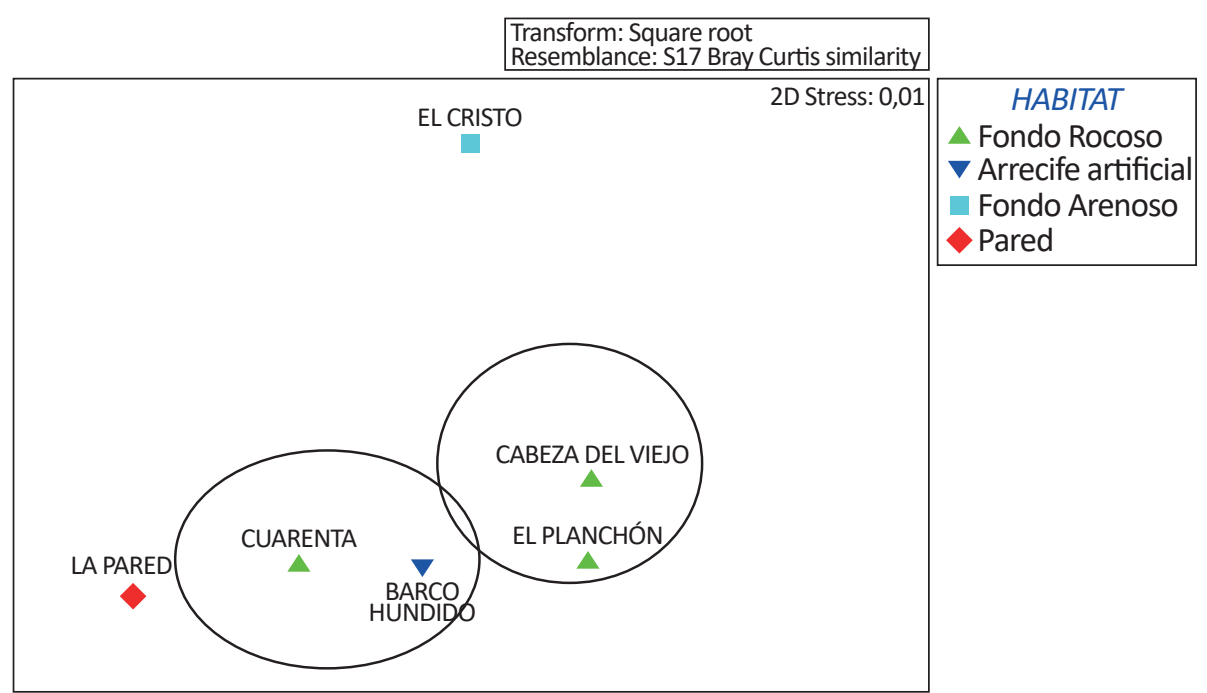

Figura 4. MDS muestra la similaridad que existe entre las abundancias de peces en siete sitios de buceo en la Reserva Marina El Pelado.

fue El Cristo caracterizado esencialmente por sus fondos arenosos (Tabla 5).

\section{Observaciones adicionales}

Durante el desarrollo de este estudio se observaron observaron diferentes actividades realizadas en los alrededores del islote como pesca, buceo y paseos en bote; se registró el uso de diferentes artes de pesca como: caña de pescar usadas desde yates (troleo), arpones usados por buzos deportivos (arponeo), compresores
('Hooka"), anzuelos y trasmallos; así también, se observó que algunas embarcaciones no usaban la boya de amarre y lanzaban sus anclas sobre los fondos rocosos y coralinos, lo cual pudo causar la fragmentación de ciertas colonias de Pocillopora observadas en los sitios Cabeza del Viejo y la Zona Protegida. Adicionalmente, se registró el uso de algunos sitios de buceo por más de dos embarcaciones al mismo tiempo por lo que de continuar esta situación en el futuro se podrían generar impactos

Tabla 5.

Índices de Diversidad de peces (Índice Shannon Wiener $\left(\mathrm{H}^{\prime}\right)$ e índice de Simpson (D)) y Equitabilidad (Índice de Pielou $\left.\left(\mathrm{J}^{\prime}\right)\right)$ presentes en los sitios de estudio.

\begin{tabular}{|c|c|c|c|c|c|}
\hline Sustrato & Sitios & $\begin{array}{l}\text { Número de } \\
\text { especies } \\
(S)\end{array}$ & $\begin{array}{c}\text { Índice } \\
\text { Shannon } \\
\text { Wiener } \\
\left(H^{\prime}\right)\end{array}$ & $\begin{array}{l}\text { Índice de } \\
\text { Simpson } \\
(D)\end{array}$ & $\begin{array}{c}\text { Equitabilidad } \\
\left(J^{\prime}\right)\end{array}$ \\
\hline \multirow{4}{*}{ Fondo Rocoso } & La Pared & 24 & 4 & 0,93 & 0,88 \\
\hline & $\begin{array}{l}\text { Cabeza del } \\
\text { Viejo }\end{array}$ & 27 & 3,55 & 0,87 & 0,75 \\
\hline & El Cuarenta & 22 & 2,54 & 0,67 & 0,57 \\
\hline & El Planchón & 12 & 2,48 & 0,77 & 0,69 \\
\hline \multirow{3}{*}{ Fondo arenoso } & $\begin{array}{l}\text { El Barco } \\
\text { Hundido }\end{array}$ & 25 & 2,95 & 0,74 & 0,63 \\
\hline & Zona Protegida & 8 & 2,73 & 0,88 & 0,91 \\
\hline & El Cristo & 8 & 1,29 & 0,42 & 0,43 \\
\hline
\end{tabular}




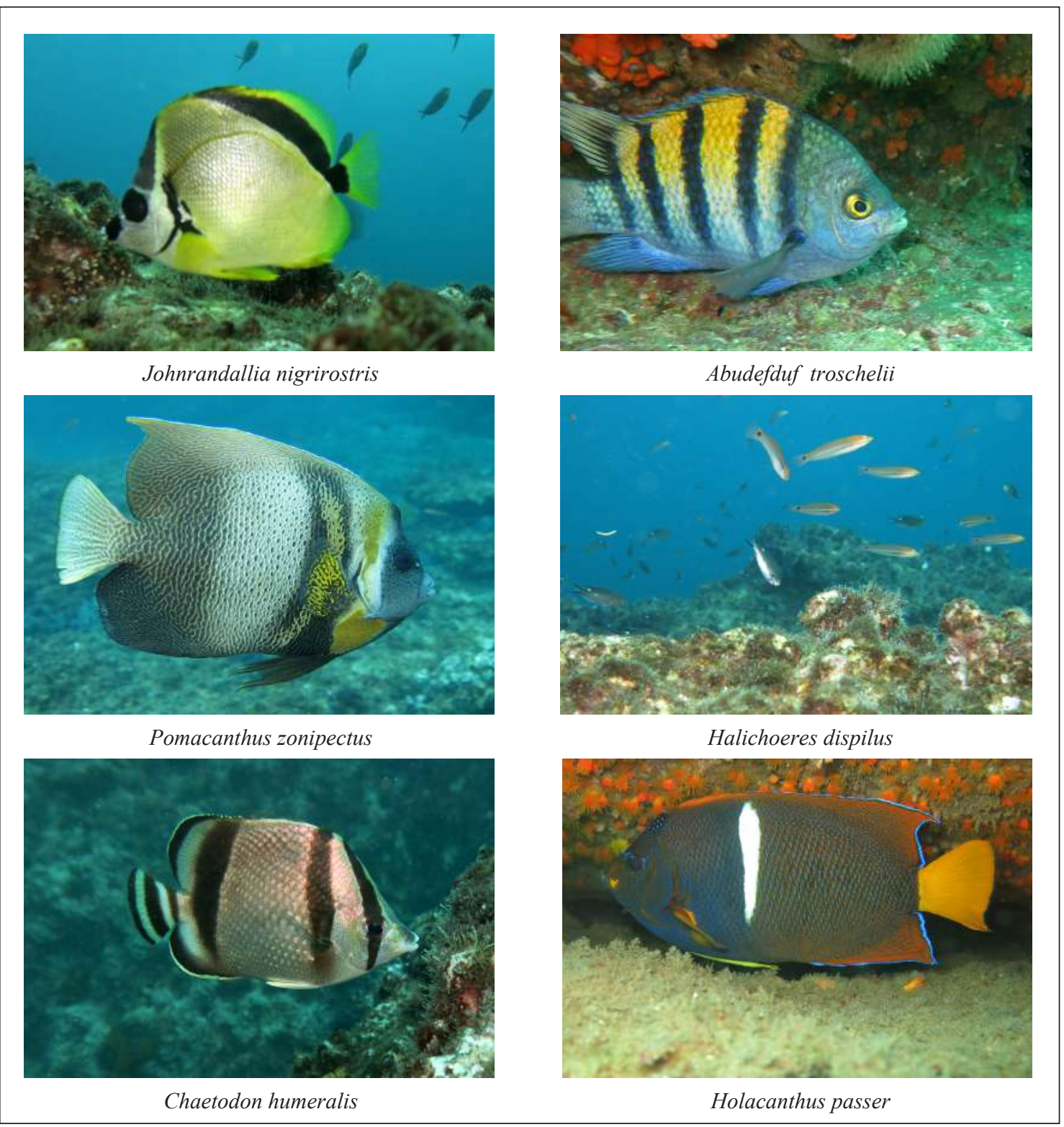

Figura 5. Principales especies de peces registrados en los sitios de buceo situados alrededor del Islote El Pelado en la Reserva Marina El Pelado.

negativos sobre las comunidades variada de organismos sésiles y peces en bentónicas. En la Figura 5 puede el área circundante al Islote El Pelado de apreciarse fotografías de algunas de las especies de peces observadas.

\section{Discusión}

Los resultados de este estudio indican la presencia de una comunidad muy la Reserva Marina con 114 especies (43 especies de organismos sésiles, 21 especies de macroinvertebrados y 50 especies de peces arrecifales). Los valores obtenidos son superiores a los registrados para los estudios realizados en el 2008 (Cárdenas, 2008) y 2012 (Rivera,

82

INVESTIGATIO No. 11, noviembre 2018,

Edición Especial, pp. 67-88,

ISSN: 1390 - 6399・ISSN-e: 2602 - 8336 
2012) esto se explica por el mayor número de sitios muestreados. Los sitios con mayor diversidad biológica fueron: La Pared, El Cuarenta y la Cabeza del Viejo caracterizados por tener predominantemente rocosos.

Se confirma el predominio de cnidarios, equinodermos y moluscos en los sitios de estudio, los mismos que cumplen importantes roles funcionales a nivel de la red alimenticia como filtradores (Cognetti et al., 2001), reguladores ecológicos (Caso, 1994) y como bioindicadores de perturbaciones ambientales (Solís-Weiss, 1982), quienes habitan en su mayoría sobre sustratos rocosos (Keen, 1971; Brusca, 1973) y en menor proporción en sustratos arenosos (Cognetti et al., 2001), constituyendo recursos cuyas abundancias se han mantenido elevadas en relación a otros taxones según los estudios realizados por Rivera en el 2012.

Se encontraron diferencias significativas sólo en el ensamblaje de macroinvertebrados móviles a nivel de hábitats, sus mayores abundancias se registraron en bajos rocosos a profundidades que oscilaron entre 8 y 30 m. Los macroinvertebrados móviles que predominaron fueron los equinodermos Phataria unifascialis, Echinometra vanbrunti y Centrostephanus coronatus, dominancia similar a la registrada para equinodermos en otras zonas del Pacífico como la costa oriental de Baja California Sur donde el poco interés comercial en este grupo (González-Medina et al., 2006) podría mantener mayor abundancia de sus poblaciones; así también los sustratos rocosos irregulares (Caso, 1994) formados por oquedades $y$ tapizados con algas podrían brindarles refugio y disponibilidad de alimento, generando condiciones apropiadas para el desarrollo de estos macroinvertebrados.

La mayor diversidad de invertebrados se registró en la Zona Protegida, La Pared y El Cuarenta, coincidiendo con los estudios realizados por Cárdenas (2008) y Rivera (2012). Esto estaría asociado principalmente con el tipo de sustrato, el cual constituye un factor ecológico determinante para la regulación de la estructura comunitaria de las comunidades bentónicas (González Medina, et al., 2006), así como por la inclinación, pendiente, rugosidad de los sustratos rocosos que generan un hábitat propicio para el asentamiento de las especies bentónicas.

Los taxones más representativos y diversos a nivel de organismos sésiles fueron los cnidarios (23 especies) conformados por comunidades de corales pétreos, corales córneos, hidrozoarios y octocorales. La alta incidencia de las colonias de octocorales; las cuales son muy representativas en el área de estudio podrían tener relación con el tipo y disponibilidad del sustrato (Kinzie \& Robert, 1973), por la capacidad de fijación en fondos con poca inclinación (Rey, 2011), influencia de la luz, poca profundidad de los bajos, régimen hidrodinámico y por la consolidación del sustrato rocoso como lo registrado en otras zonas tropicales (Alcolado, 1981). Las familias predominantes de estas colonias de octocorales fueron Gorgoniidae y Plexauridae. De igual 
forma la abundancia de Plexauridae en este estudio sugiere que es abundante en la mayoría de los sitios evaluados alrededor del Islote El Pelado, siendo Muricea appressa la especie más frecuentemente observada.

Debido a la alta diversidad macrobentónica encontrada, los sitios El Cuarenta, La Pared y la Cabeza del Viejo, deben ser considerados prioritarios para el control y conservación de la biodiversidad en esta reserva, ya que habitan especies sensibles como: Anthipathes galapagensis, Myriopathes panamensis, Pocillopora damicornis, P.capitata y P. elegans las cuales están registradas en el apéndice II de CITES y en la lista roja de IUCN, las mismas que son frágiles ante uso inadecuado de los bajos donde ellas habitan. De estas especies $A$. galapagensis y $M$. panamensis son comunes en bajos más profundos como La Pared (superiores a $20 \mathrm{~m}$ ) y contrariamente los pocilóporos se encuentran con mayor frecuencia en bajos más someros $(5-13 \mathrm{~m})$. Por tanto, se sugiere que dichos sitios tengan mejor supervisión de los guías de buceo y se controle el uso de los sitios según la fragilidad de la biota bentónica, el nivel de entrenamiento y experiencia de los buzos, evitando las inmersiones iniciales de buzos principiantes en las zonas poco profundas donde existe mayor influencia del oleaje y la tensión hidrodinámica para minimizar la fragmentación de corales.

Entre las especies de invertebrados de importancia comercial que se registraron en la reserva fueron: Octopus sp., Spondylus calcifer, Hexaplex princeps,
Hexaplex spp. e Isostichopus fuscus. Además, se encontró el octocoral invasor conocido como Carijoa riseii en el bajo El Cuarenta, reportado por Rivera \& Martínez (2011) en la Reserva Marina Galera-San Francisco (Esmeraldas) en la costa norte del país, lo que evidencia su expansión hacia la costa centro- sur, esto resulta preocupante ya que esta especie forma agregaciones mono-específicas muy densas que compiten por espacio, luz y alimento con especies sésiles como los corales negros como lo sucedido en las costas de Hawaii (Kahng \& Grigg, 2005).

A nivel de peces, los ensamblajes bióticos presentaron diferencias significativas entre los diferentes hábitats, los mayores registros de abundancia, fueron representados por Serranidae, Pomacentridae, Haemulidae, Labridae y Tetradontidae, siendo las especies más abundantes Paranthias colonus, Halichoeres dispilus y Johnrandallia nigrirostris. El $90 \%$ de las especies registradas en este estudio, correspondieron a especies de peces ornamentales, mientras que el $10 \%$ correspondieron a especies comerciales pertenecientes a las familias Haemulidae (Anisostremus interruptus), Serranidae (Epinephelus labriformis, Cratinus agassizii y Cratinus sp.) y Lutjanidae (Lutjanus argentiventris y Lutjanus sp.). Esta disminución podría estar asociada a que estas especies son capturadas en las faenas de pescadores artesanales de la zona.

Otras especies de peces como las rayas Urobatis halleri y Rhinobatos 
phrali fueron registradas en este estudio, siendo propias de fondos arenosos, esto coincide con las observaciones realizadas por Feijó en la Zona Protegida y la Cabeza del Viejo durante el 2012 y 2013 (Feijó, 2013), así también la presencia de la Manta birrostris observada en el bajo La Pared, lo cual maximiza el atractivo turístico de los bajos de buceo en esta área protegida.

Las especies más representativas del área de estudio fueron: Scarus perrico, Chaetodon humeralis y Johnrandallia nigrirostris observándose gran cantidad de individuos en las zonas rocosas con crecimiento algal, especialmente conformadas por rodofitas. Otro hábitat importante por la abundancia de especies fue el Barco Hundido, donde se refugiaban gran variedad de especies por lo que se considera que este arrecife artificial, estaría cumpliendo su objetivo como agregador biológico en zonas empobrecidas como los arenales donde fue sumergido en el 2013 (Ministerio del Ambiente, 2013).

El turismo de buceo no controlado en zonas coralinas en aguas poco profundas, podría causar daños irreversibles, así como las visitas simultánea de buzos y de embarcaciones, posibles residuos de combustibles de las embarcaciones y principalmente el uso de redes de fondo que aún se usan en la pesca artesanal en los alrededores del Islote El Pelado podrían en el corto y mediano plazo degradar este ecosistema marino y sobrepasar su capacidad de resiliencia. Se sugiere que las autoridades ambientales implementen medidas preventivas y de control de la contaminación de los sitios de buceo situados alrededor del Islote El Pelado y la Bahía de Ayangue, así también hacer el seguimiento $\mathrm{y}$ monitoreo de las actividades antrópicas para evaluar el impacto sobre las zonas arrecifales y ejecutar un programa de educación ambiental en colaboración con centros e instructores de buceo, escuelas locales y sector turístico que operan en esta reserva, para concientizar a los usuarios sobre su rol en la conservación de los recursos marinos del área.

Es importante dar continuidad a los monitoreos biológicos e incorporar la evaluación de variables ambientales que permitan medir el efecto de actividades antrópicas como el turismo y las actividades pesqueras artesanales que puedan llegar a tener sobre las diferentes comunidades macrobentónicas. Así también se debe prohibir el expendio de especies como Pocillopora spp., Phataria unifascialis e Hippocampus sp., en la reserva marina y gestionar proyectos productivos y de conservación de los recursos marinos con la participación activa de pescadores artesanales y comuneros de Ayangue, Palmar y San Pedro para disminuir las presiones al ambiente y fomentar medios de subsistencia compatibles con el manejo sustentable de la Reserva Marina El Pelado.

\section{Agradecimientos}

Agradecemos a los biólogos Fernando Aguilar y Walter Mendívez del Instituto Nacional de Pesca quienes colaboraron en la identificación parcial de peces. Los autores agradecen el apoyo financiero del Fondo Mundial para el Medio Ambiente (por sus 
siglas en inglés (GEF) en el marco del proyecto "Facilitación de financiamiento para negocios basados en la biodiversidad y apoyo de actividades de desarrollo de mercados en la región Andina" implementado por la Corporación Andina de Fomento (CAF) y Corpei (iniciativa Biocomercio Andino GEF-CAF). Apreciamos los comentarios y sugerencias de los revisores anónimos y a los investigadores Luis Troccoli y Nikita Gaibor quienes contribuyeron al mejoramiento del manuscrito.

\section{Referencias}

Aguilar, F. W. Revelo, D. Chicaiza, W. Mendívez y D. Hill.(2009). Estado poblacional del pepino de mar Isostichopus fuscus (Ludwing, 1875) en las provincias de Santa Elena y sur de Manabi. Guayaquil.

Aguilar, W. Revelo, L. Delgado y J. F. Vitar. (2010). Reporte biológico de la concha Spondylus (Spondylus calcifer y S. princeps) en la zona de Ayangue-Santa Elena. Ecuador: Instituto Nacional de Pesca-Proceso IRBA.

Alcolado, P.M. (1981). Zonación de los octocorales someros de Cuba y su posible uso como indicadores comparativos de tensión hidrodinámica sobre los organismos del bentos. Informe Científico Técnico. La Habana: Instituto de Oceanología.

Allen, G. R.\& D. R. Robertson (2006). Shorefishes of the tropical eastern Pacific: an information system. Version 2.0. Balboa: Smithsonian Tropical Research Institute.

Boothroyd, J., Ayón, H., Robadue, D., Vásconez, J., \& Noboa, R. (1994). Características de la línea costera del Ecuador $y$ recomendaciones para su manejo. Programa de Manejo de Recursos Costeros. Reporte Técnico 2076. Ecuador.
Brusca, R. (1973). A hanbook to the Common Intertidal Invertebrates of the Gulf of California. Tucson: Arizona Press.

Cárdenas, M. (2008). Informe del curso de capacitaciones de métodos de muestreo submareal de peces e invertebrados marinos para estudios de biodiversidad. Conservación Internacional y BIOELITE. Guayaquil, Ecuador.

Caso, ME. (1994). Estudio morfológico, taxonómico, ecológico y distribución geográfica de los asteroideos recolectados durante las campañas oceanográficas Cortés 1, 2, 3. Anales del. Instituto de Ciencias del Mar y Limnología, 22, 101-119.

CITES. (2018). Convention on International Trade in Endangered Species of Wild Fauna and Flora. Recuperado de: https://www.cites.org/eng/.2018/06/20.

Clarke, K., R., \& Warwick, R. M., (1994). Change in marine communities: an approach to statistical analysis and interpretation. Plymouth: Natural Environment Research Council.

Clarke, K., R., \& Warwick, R., M., (2001). Change in marine communities: an approach to statistical analysis and interpretation. Plymouth: Primer-E Ltd.

Cognetti,G,M. \& G. Magazzu. ( 2001). Biología marina. España: Editorial Ariel.

Cruz, M., Gabor, N., Mora, E., Jiménez, R., \& Mair, J. (2003). Lo conocido y desconocido de la Biodiversidad Marina en el Ecuador (Continental e Insular). Gayana (Concepción), 67(2), 232-260.

Edgar, G. J., Banks, S. A., Bessudo, S., Cortés, J., Guzmán, H. M., Henderson, S., \& Zapata, F. A. (2011). Variation in reef fish and invertebrate communities with level of protection from fishing across the Eastern Tropical Pacific seascape. Global Ecology and Biogeography, 20(5), 730-743. 
Feijoó, J. (2013). Identificación, distribución y elaboración de un catálogo de batoideos (rayas, guitarras y torpedos) que habitan en la Reserva Marina Islote El Pelado. Tesis de grado. Universidad Estatal Península de Santa Elena. Ecuador.

González-Medina, F. J., Holguin-Quiñones, O. E., \& De la Cruz-Agüero, G. (2006). Variación espaciotemporal de algunos macroinvertebrados (Gastropoda, Bivalvia y Echinodermata) de fondos someros del archipiélago Espíritu Santo, Baja California Sur, México. Ciencias Marinas, 32(1A), 33-44.

Glynn, P. W., Maté, J. L., Baker, A. C., \& Calderón, M. O. (2001). Coral bleaching and mortality in Panama and Ecuador during the 1997-1998 El Niño-Southern Oscillation event: spatial/temporal patterns and comparisons with the 1982-1983 event. Bulletin of Marine Science, 69(1), 79-109.

Hickman, C. \& Rojas Lizana, I. (1998). Guía de campo sobre estrellas de mar y otros equinodermos de Galápagos. Lexington: Sugar Spring Press.

Hickman, C. \& Y. Finet. (1999). A field guide to Marine Molluscs of Galápagos. Lexington: Sugar Spring Press

Hickman, C., \& Todd, Z. (2000). Guía de campo de los Crustáceos de Galápagos. Serie Vida Marina de Galápagos. Lexington: Sugar Spring Press

Hickman, C. P. (2008). A Field Guide to Corals and other Radiates of Galapagos. Galapagos Marine Life Series. Lexington: Sugar Spring Press

Humann, P. (1993). Reef fish identification: Galapagos. Florida: New World Publications.

IUCN. (2018). The IUCN Red List of the Threatened species. Recuperado de: http://www.iucnredlist.org/details/180373 /0.
Kahng, S. E., \& Grigg, R. W. (2005). Impact of an alien octocoral, Carijoa riisei, on black corals in Hawaii. Coral Reefs, 24(4), 556-562.

Keen, M. (1971). Sea Shells of Tropical West America. (2nd. Edition ed.). California: Stanford University.

Kinzie, I. I. I., \& Robert, A. (1973). Coral Reef project-papers in memory of Dr. Thomas F. Goreau. 5. The zonation of West Indian gorgonians. Bulletin of Marine Science, 23(1), 93-155.

Ministerio del Ambiente del Ecuador. (2013). Fondeo de Lancha Rigel aportará a creación de arrecifes artificiales en Reserva Marina El Pelado. Recuperado de:

http://www.ambiente.gob.ec/fondeo-de-lan cha-rigel-en-reserva-marina-el-pelado/

Ministerio del Ambiente. (2014). Plan de Manejo de la Reserva Marina El Pelado. Quito: Ministerio del Ambiente del Ecuador.

Mora, E., V. Jurado y W. Mendívez. (2010). Diversidad de macroinvertebrados en la plataforma continental de Ecuador. Revista Ciencias del Mar y Limnología, 4(2), 101-106.

Morris, P. A. (1966). A Field Guide to Pacific Coast Shells: Including shells of Hawaii and the Gulf of California. Sponsored by the National Audubon Society and National Wildlife Federation (Second Edition). Boston: Houghton Mifflin.

Perrone, A.,D. Cajiao y M. Burgos. (2009). Turismo de naturaleza en la zona marino costera del Ecuador continental. Conservación Internacional Ecuador, Ministerio del Ambiente del Ecuador, Ministerio de Turismo del Ecuador. Guayaquil, Ecuador.

Rivera, F \& Martínez. P. (2011). Consolidación de la reserva Marina Galera San Francisco. Reporte Técnico del monitoreo Submareal. 
Rivera F. (2012). Levantamiento de la línea base biológica en el perfil costero del Ecuador Continental. Informe técnico. Subsecretaria de Gestión Marino Costera. Ecuador.

Solıs-Weiss, V. (1982). Aspectos ecológicos de la contaminación orgánica sobre el macrobentos de las cuencas de sedimentación en la bahı de Marsella Francia. Anales del Instituto de Ciencias del Mar y Limnología, 9(1), 19-44.

Suárez, X. (2013). Caracterización de atractivos naturales de los cuatro sitios de buceo más visitados en el Islote El Pelado y la propuesta de una guía de buenas prácticas de buceo. Tesis de grado. Universidad de Especialidades Espíritu Santo. Guayaquil, Ecuador.

Wilber, D. H., \& Clarke, D. G. (1998). Estimating secondary production and benthic consumption in monitoring studies: a case study of the impacts of dredged material disposal in Galveston Bay, Texas. Estuaries, 21(2), 230-245.

Zar, J.H. (1996). Biostatistical Analysis. Tercera Edición. New Jersey: Practice Hall. 